\title{
Falls and Fractures in Patients with Parkinson's Disease-Related Psychosis Treated with Pimavanserin vs Atypical Antipsychotics: A Cohort Study
}

\author{
J. Bradley Layton ${ }^{1}$ (D) Joan Forns ${ }^{2}$ (D) Mary Ellen Turner ${ }^{3} \cdot$ Colleen Dempsey $^{3} \cdot$ Jennifer L. Bartsch ${ }^{1}$ (1) .

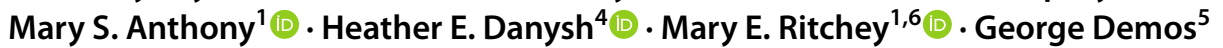

Accepted: 6 October 2021 / Published online: 30 October 2021

(c) The Author(s) 2021

\begin{abstract}
Background Parkinson's disease-related psychosis increases patients' risk of falls. Pimavanserin is an atypical antipsychotic approved in the USA in 2016 for the treatment of hallucinations and delusions associated with Parkinson's disease-related psychosis.

Objective We aimed to compare the risk of falls/fractures among patients with Parkinson's disease-related psychosis treated with pimavanserin vs other atypical antipsychotics.

Patients and Methods We identified a cohort of patients with Parkinson's disease-related psychosis aged $\geq 40$ years initiating either pimavanserin or a comparator antipsychotic (clozapine, quetiapine, risperidone, olanzapine, aripiprazole, brexpiprazole) in US commercial insurance and supplementary Medicare claims (2015-2019). Comparators were propensity score matched 2:1 with pimavanserin initiators; incidence rates of falls/fractures were compared using incidence rate ratios (IRRs) and $95 \%$ confidence intervals (CIs).

Results We identified 112 eligible pimavanserin initiators and 982 comparators. Pimavanserin initiators were younger and had fewer severe comorbidities, indicators of impairment, and healthcare encounters, though they had higher Parkinson's disease medication use. The crude incidence rates [cases/100 person-years] $(95 \% \mathrm{CI})$ for composite falls/fractures were 17.8 (7.7-35.0) for pimavanserin and 40.8 (35.0-47.4) for comparators. Matching retained 108 pimavanserin initiators and 216 comparators - all characteristics were well balanced after matching — with a matched IRR (pimavanserin vs comparator) of 0.71 (95\% CI 0.27-1.67). Sensitivity analysis IRR estimates were consistently below 1.00 , with a sensitivity analysis not requiring a diagnosis of psychosis resulting in an IRR estimate of 0.55 (95\% CI 0.34-0.86).

Conclusions The results of this study do not suggest an increase in the risk of falls or fractures associated with pimavanserin compared with other antipsychotics in patients with Parkinson's disease-related psychosis. Sensitivity analyses suggest a decreased risk.
\end{abstract}

\section{Introduction}

Patients with Parkinson's disease (PD) are at increased risk for falls and fractures [1], with many features of PD being predisposing risk factors for falls and fractures, including cognitive and motor impairment, postural instability, hypotension, unsteady gait, bradykinesia, rigidity, and frailty. Additionally, psychosis may occur in up to $75 \%$ of patients with PD [2], particularly in more advanced stages of the disease, and patients with PD-related psychosis (PDP) are

J. Bradley Layton

jblayton@rti.org

Extended author information available on the last page of the article

\section{Key Points}

Patients with Parkinson's disease psychosis have a high risk of fractures and falls requiring medical attention.

Patients with Parkinson's disease psychosis being treated with pimavanserin did not have an increased risk of falls compared with patients taking other antipsychotics.

Sensitivity analyses suggested a decreased risk of falls or fractures associated with pimavanserin compared with patients taking other antipsychotics. 
at even higher risks for falls and fractures than patients with PD without psychosis [3].

Pimavanserin is a selective serotonin inverse agonist preferentially targeting 5-hydroxy-tryptamine- $2 \mathrm{~A}$ receptors that was approved in the USA in 2016 for the treatment of hallucinations and delusions associated with PDP [4]. Later that year, the US Food and Drug Administration introduced a labeling update for antipsychotic medications that required a warning of falls in patients treated with antipsychotics, especially for patients with potentially exacerbating conditions or medication use. Although pimavanserin was not included in this class warning, falls are included in the US label of pimavanserin as part of the postmarketing experience [4]. To better understand the risk of falls and fractures in users of pimavanserin relative to other antipsychotic treatments, we conducted an observational study in a large, US-based insurance claims database to compare the risk of falls and fractures among patients with PDP initiating pimavanserin vs those initiating other atypical antipsychotics.

\section{Methods}

\subsection{Data Source}

This study was conducted using two of the IBM MarketScan Research Databases (Copyright 2019 IBM Inc., all rights reserved): Commercial Claims and Encounters, and Medicare Supplemental and Coordination of Benefits. These US-based commercial insurance databases contain information about diagnoses, procedures, and pharmacy-dispensed medications from insurance billing data for employees, their spouses, and their dependents with employer-based commercial insurance from large employers across the USA and retirees with employerbased Medicare supplementary insurance. Both International Classification of Diseases, Ninth Revision, Clinical Modification (ICD-9-CM) and International Classification of Diseases, Tenth Revision, Clinical Modification (ICD10-CM) diagnosis coding systems were used at various points during the study period. Diagnosis-based code lists for study characteristics were developed using either ICD-9-CM or ICD-10-CM codes and then were mapped to the other system with General Equivalence Mapping [5]; mapped code lists were manually reviewed to ensure conceptual consistency after mapping [6].

\subsection{Population}

We identified patients aged $\geq 40$ years at their first pharmacy dispensing claims for pimavanserin or another atypical antipsychotic (clozapine, quetiapine, risperidone, olanzapine, aripiprazole, brexpiprazole) occurring between 1 May 2015, and 31 December 2019. The medication initiation date became the study index date (Fig. 1). To identify first initiation of either pimavanserin or a comparator antipsychotic, all available baseline information before the index date for each patient was evaluated to identify any prior antipsychotic use; patients with prior use of any atypical or conventional antipsychotic medication were excluded, as were patients who received more than one different study drug on the index date. Medication initiators were also required to have a minimum of 180 days of baseline enrollment before and including the index date, although if $>180$ days of baseline enrollment information was available for a patient, all of the available baseline data were used to define patient characteristics (Fig. 1). There are no specific diagnosis codes for PDP; therefore, PD and psychosis were identified separately, and patients were required to have recorded diagnoses of both PD and psychosis at any point before or on the index date. We did not require specific ordering of the $\mathrm{PD}$ and psychosis diagnoses.

We identified psychosis in patient records using diagnosis codes for delusions, hallucinations, psychosis, or paranoia in the inpatient or outpatient setting in any diagnosis position (Table S1 of the Electronic Supplementary Material [ESM]). Parkinson's disease diagnoses were identified in patient records without regard to new-onset or existing disease. A diagnosis of PD required a patient to meet any of the following three criteria fully before the index date: (1) one inpatient claim with a diagnosis of PD in any position (Table S2 of the ESM); (2) two outpatient claims with diagnoses of PD in any position, separated by at least 30 days but within 365 days; and (3) at least two pharmacy prescription dispensing claims for a PD-related medication (levodopa-carbidopa, anticholinergics, dopamine agonists, monoamine oxidase B inhibitors, catechol$O$-methyltransferase inhibitors, amantadine, or istradefylline) within 6 months before or after an outpatient claim with a PD diagnosis code in any position. For criterion 3 , the medication and diagnosis claims were permitted to occur on the same date; two medication claims on the same date were permitted only if they were for different medications.

We additionally excluded patients with diagnoses of bipolar disorder, schizophrenic disorders, or Huntington's disease before the index date to avoid disease misclassification, as these conditions may also be treated with antipsychotics. Additionally, patients were excluded if they had diagnoses of pathologic fracture that may have resulted from conditions such as cancer, infection, osteomalacia, and Paget's disease before the index date.

Two sensitivity analyses were performed to observe the robustness of the results after altering the study's inclusion/exclusion criteria. All patients in the primary analysis 


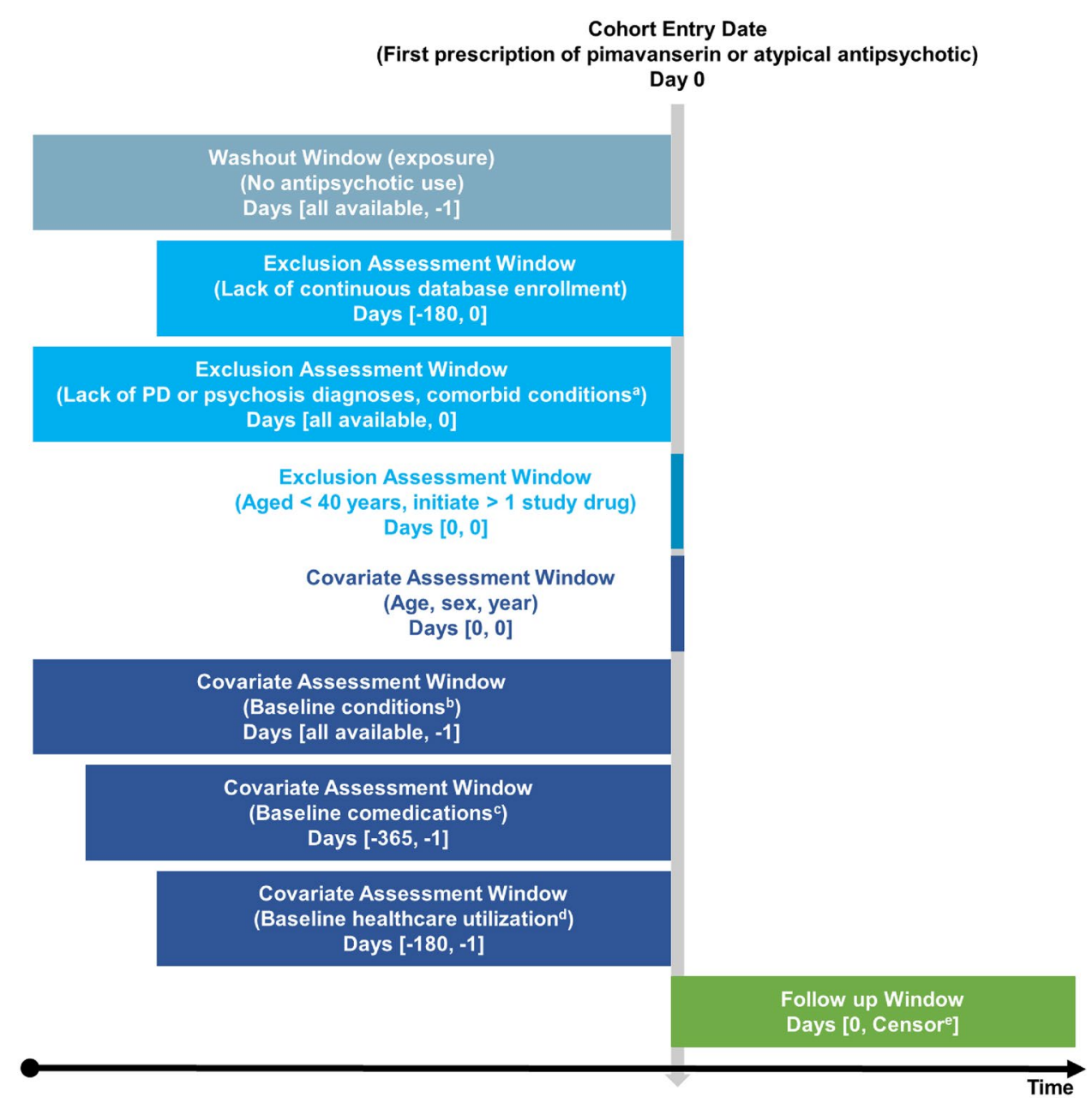

Fig. 1 Identification of eligible pimavanserin or comparator atypical antipsychotic initiators for inclusion in the study cohort. Note: The duration of each assessment window is given in days relative to the cohort entry date (day 0). Square brackets indicate the window is inclusive of the stated date. Similar sets of criteria (e.g., washout windows, exclusion assessment windows, covariate assessment windows, follow-up windows) are shown in the same color. Figure template available at www.repeatinitiative.org. $P D$ Parkinson's disease. ${ }^{a}$ Comorbid conditions that were exclusion criteria were bipolar disorder, schizophrenic disorders, Huntington's disease, and pathologic fracture. ${ }^{b}$ Baseline conditions included wheelchair use, ambulance transport or life support, bladder dysfunction, coagulopathy, home oxygen, paralysis, dementia, cancer screening, heart failure, lipid abnormality, vertigo, difficulty walking, podiatric care, rehabilitation services, arthritis, skin ulcer, sepsis, stroke/brain injury, weakness, diabetes mellitus complications, home hospital bed, myocardial infarction, peripheral vascular disease, chronic obstructive pulmonary disease, peptic ulcer disease, liver disease, diabetes mellitus, hemiplegia, chronic kidney disease, tumor, leukemia, lymphoma, human immunodeficiency virus/acquired immune deficiency syndrome,

met the strict criteria for a PD diagnosis, described earlier, but the first sensitivity analysis also excluded patients with at least one claim with a diagnosis code for secondary PD, including drug-induced or vascular PD, in any setting to remove patients potentially misclassified as having PD. delirium, osteoporosis, multiple sclerosis, celiac disease, Crohn's disease, ulcerative colitis, ankylosing spondylitis, depression, hyperthyroidism, Cushing's syndrome, hyperparathyroidism, vitamin D deficiency, malnutrition, impaired vision, and orthostatic hypotension. ${ }^{c}$ Baseline comedications included osteoporosis treatment, androgen deprivation therapy, nonsteroidal anti-inflammatory drugs, cyclooxygenase-2 inhibitors, systemic glucocorticoids, enzyme-inducing anticonvulsants, thiazolidinediones, benzodiazepines, sedatives, digoxin, diuretics, anticholinesterase inhibitors, antidepressants, and Parkinson's disease drugs (levodopa-carbidopa, anticholinergics, dopamine agonists, monoamine oxidase $\mathrm{B}$ inhibitors, catechol- $O$-methyltransferase inhibitors, amantadine, and istradefylline). ${ }^{\mathrm{d}}$ Baseline healthcare utilization included number of hospitalizations and number of emergency department visits. ${ }^{\mathrm{e}}$ Earliest occurrence of administrative study end (31 December 2019); disenrollment from the MarketScan databases; pathological fracture that may have resulted from conditions such as cancer, infection, osteomalacia, and Paget's disease; diagnosis of bipolar disorder, schizophrenic disorders, or Huntington's disease; discontinuation of index medication; and initiation of a different study antipsychotic other than the index treatment

Additionally, the primary analysis required all included patients to have a recorded diagnosis of psychosis; however, as psychosis symptoms may not be consistently coded in claims data [7-9], the second sensitivity analysis did not require patients to have a psychosis diagnosis. 


\subsection{Treatment Assessment}

The treatment group consisted of patients with PDP initiating antipsychotic treatment with pimavanserin, and the comparator group consisted of patients with PDP initiating antipsychotic treatment with one of the study atypical antipsychotics (clozapine, quetiapine, risperidone, olanzapine, aripiprazole, brexpiprazole). Continuous exposure to the index antipsychotic treatment was defined by assuming that the duration of each dispensed prescription was equal to the prescription's days' supply as indicated on the claim. A 30-day supply was imputed if the claim's days' supply information was missing or implausible $(<1$ day or $>90$ days). At the end of the prescription's days' supply, a grace period equal to $50 \%$ of the days' supply began. If the patient refilled the prescription during the grace period, the continuous exposure period was extended by the duration of the new prescription from the date of the new prescription. If the patient did not refill the medication by the end of the grace period, the patient was considered to have discontinued the medication on the last day of the grace period. If a patient refilled the prescription before the end of the previous prescription's days' supply (i.e., before the start of the grace period), overlaps in days' supply between consecutive prescriptions were added to the end of a continuous exposure period (i.e., stockpiled).

Patients were followed from the date of drug initiation and were censored at the first occurrence of any of the following: administrative study end (31 December 2019), disenrollment from the database, diagnosis of one of the original study exclusion criteria (pathological fracture, bipolar disorder, schizophrenic disorders, or Huntington's disease), discontinuation of index medication, and initiation of a different study antipsychotic other than the index treatment.

\subsection{Outcome Assessment}

We identified falls and fractures occurring during follow-up, and patients could experience multiple fall and/or fracture outcome events. As in previous assessments of this population $[1,3]$, falls and fractures were assessed as both composite and separate outcomes in the following groupings: (1) composite falls/fractures, (2) falls, (3) any fracture, and (4) site-specific fractures of key interest (femur, hip, pelvis, upper limb, vertebrae).

Falls were identified from diagnosis codes for accidental falls occurring in any setting or diagnosis position (Table S3 of the ESM). To reduce the potential for double counting events if a single fall resulted in multiple encounters or claims, all additional codes for falls occurring within 7 days of the initial code were considered part of the same event, and patients did not contribute at-risk person-time during this 7-day period; the date of the first diagnosis during this period was assigned as the fall event date. The occurrence of falls before the index date was evaluated to distinguish new falls during the follow-up from continuing care for falls that occurred before the beginning of the follow-up; 30 days of pre-index time was used to account for multiple consecutive 7-day periods to define recurrent falls, if required.

Fracture outcome events were categorized and evaluated both as overall "any fracture" and also as individual site-specific fractures of interest. Fracture events were defined using inpatient and outpatient diagnosis codes in any diagnostic position (Table S3 of the ESM), and each fracture diagnosis was categorized by body site (skull, vertebrae, trunk, upper limb, hand/wrist, pelvis, hip, femur, lower leg, or foot/ ankle). To ensure identification of new fractures, diagnosis codes were required to be paired with a site-specific fracture repair procedure code occurring within 7 days before or after the diagnosis code, with the earlier of the diagnosis and repair code dates assigned as the fracture event date. To avoid double counting return visits for the same fracture, all subsequent fracture diagnoses occurring at the same site within 1 year after the initial site-specific fracture were considered part of the same event, and individuals did not contribute at-risk person-time for fractures at the same site during that 1-year period [1]. Site-specific fractures in the year before the index date were evaluated to accurately define patients' at-risk status at the beginning of follow-up. Site-specific fractures were identified first and then collapsed into the overall "any fracture" category. As a single incident may result in fractures at multiple sites, fractures at different sites occurring within 7 days of each other were considered part of the same event for the "any fracture" outcome. If this occurred, the date of the earliest fracture was assigned as the "any fracture" event date, and the patient was not considered at risk for 7 days after that date. However, a patient could experience a site-specific fracture (e.g., upper arm fracture) and, although the patient would not be at risk for fractures at the same site for 1 year, the patient would continue to be at risk for fractures at other sites (e.g., hip fracture) after a 7-day not-at-risk period.

For the composite falls/fractures outcome, the fall and fracture events described earlier were combined into one composite measure. Patients experiencing either a fall or site-specific fracture were categorized as having a composite event on the date of the qualifying claim. The patient was not considered at risk for a recurrence of the same event type (fall or site-specific fracture) until after the event-specific, not-at-risk period ( 7 days for falls, 1 year for site-specific fractures), although the patient continued to be at risk for different events, which would be considered a composite event during that period (e.g., a patient experiencing a hip fracture would be at risk for a fall or other site-specific fracture after 7 days but would not be at risk for another hip fracture for 1 year). 


\subsection{Covariates}

We identified patient characteristics using all diagnosis, procedure, and medication dispensing information available before a patient's index date for use in descriptive analyses and as covariates in the propensity score models. Characteristics included patient demographics (age, sex), frailty indicators [10, 11], components of the Charlson Comorbidity Index [12, 13], risk factors of falls or fractures, comedications (assessed using a maximum of 1 year of baseline data before the index date), and measures of healthcare utilization (hospitalizations and emergency department visits) in the 180 days before the index date (Fig. 1).

\subsection{Statistical Approach}

Baseline characteristics were compared between patients with PDP initiating pimavanserin and those initiating other atypical antipsychotics. Counts and proportions of patients with each covariate were presented for categorical variables, while continuous variables were summarized using means and standard deviations (SDs). Absolute standardized differences were used to quantify imbalances in baseline covariate distributions between treatment groups [14] in the unmatched and matched populations, with standardized difference values closer to 0 indicating better covariate balance.

Propensity score matching was used to control for baseline confounders. The probability of being treated with pimavanserin as opposed to a comparator antipsychotic was estimated using multivariable logistic regression including a priori-identified covariates; comorbidities and comedication variables were included as binary terms, and age was included with linear, quadratic, and cubic terms. Covariates were not included in the model if they were not present in at least one patient in both treatment groups. After propensity scores were estimated, comparator antipsychotic initiators were matched to pimavanserin initiators in a 2:1 fixed matching ratio without replacement by using a greedy nearest neighbor, a 5-digit to 1-digit matching algorithm [15], and with a maximum caliper of 0.2 times the SD of the estimated logit of the propensity score [16].

Within the unmatched and matched cohorts, we estimated crude incidence rates by treatment group for the fall, fracture, and composite outcomes as the number of cases divided by the duration of follow-up with accompanying 95\% confidence intervals (CIs) [17]. For comparisons of the incidence rates across treatment groups, incidence rate ratios (IRRs) and 95\% CIs were estimated [18] in the unmatched and matched cohorts. Incidence rate ratios were estimated across the entire follow-up period and for each year increment of follow-up (0-1 year, > 1-2 years, $>2$ years).

All analyses were performed using SAS version 9.4 or higher (SAS Institute, Inc., Cary, NC, USA). This analysis of deidentified data was determined to not constitute research of human subjects by the Institutional Review Board of RTI International.

\section{Results}

We identified 1094 eligible patients diagnosed with PDP who initiated antipsychotic treatment. Of these included patients, $112(10 \%)$ were initiators of pimavanserin, and $982(90 \%)$ were initiators of a comparator antipsychotic (Fig. 2); the comparator group consisted primarily of quetiapine users $(79 \%)$, followed by risperidone $(12 \%)$, olanzapine $(6 \%)$, aripiprazole $(3 \%)$, and clozapine and brexpiprazole ( $<1 \%$ each). Users of low-dose quetiapine were by far the largest proportion of the comparator group ( $90 \%$ of the quetiapine users never had dispensed strengths of greater than $50 \mathrm{mg}$ during the follow-up period). The pimavanserin treatment group was $31 \%$ female and had a mean (SD) age of 76.1 (8.1) years. The comparator antipsychotic group was $38 \%$ female, with a mean (SD) age of $78.4(9.8)$ years.

There were differences in patient characteristics between the unmatched treatment groups (Fig. 3). Pimavanserin initiators were younger and had fewer severe comorbidities and indicators of major impairment, including delirium (77\% vs $85 \%$ ), use of ambulance/life support services (61\% vs $77 \%$ ), stroke/brain injury ( $45 \%$ vs $58 \%$ ), heart failure ( $34 \%$ vs $45 \%$ ), paralysis ( $6 \%$ vs $15 \%)$, and hemiplegia ( $5 \%$ vs $11 \%$ ), and had fewer hospitalizations (mean, 0.3 vs 0.6 ) and emergency department visits (mean, 1.2 vs 2.0 ) (Table 1). Pimavanserin initiators also had a notably lower prevalence of depression diagnoses (51\% vs $63 \%$ ) and a lower use of antidepressants (49\% vs $61 \%$ ). However, pimavanserin initiators had a higher prevalence of PD treatments than comparator antipsychotic initiators, including the use of PD medications (96\% vs 83\%) and anticholinesterase inhibitors (51\% vs 39\%).

In the crude unmatched cohort, 181 composite fall/ fracture events were identified during 469 person-years of follow-up (45 in the pimavanserin group and 424 in the comparator group). The majority of identified outcomes were falls (Table 2; Table S4 of the ESM). The crude incidence rates of composite falls/fractures (events per 100 person-years) were 17.8 (95\% CI 7.7-35.0) among pimavanserin initiators and 40.8 (95\% CI 35.0-47.4) among comparator antipsychotic initiators for a crude IRR of 0.44 (95\% CI 0.19-0.88) when comparing pimavanserin with comparator antipsychotics.

The propensity score distributions demonstrated a good overlap between the treatment groups (Fig. 4). After propensity score matching, almost all pimavanserin initiators were successfully matched to two comparators, with 108 


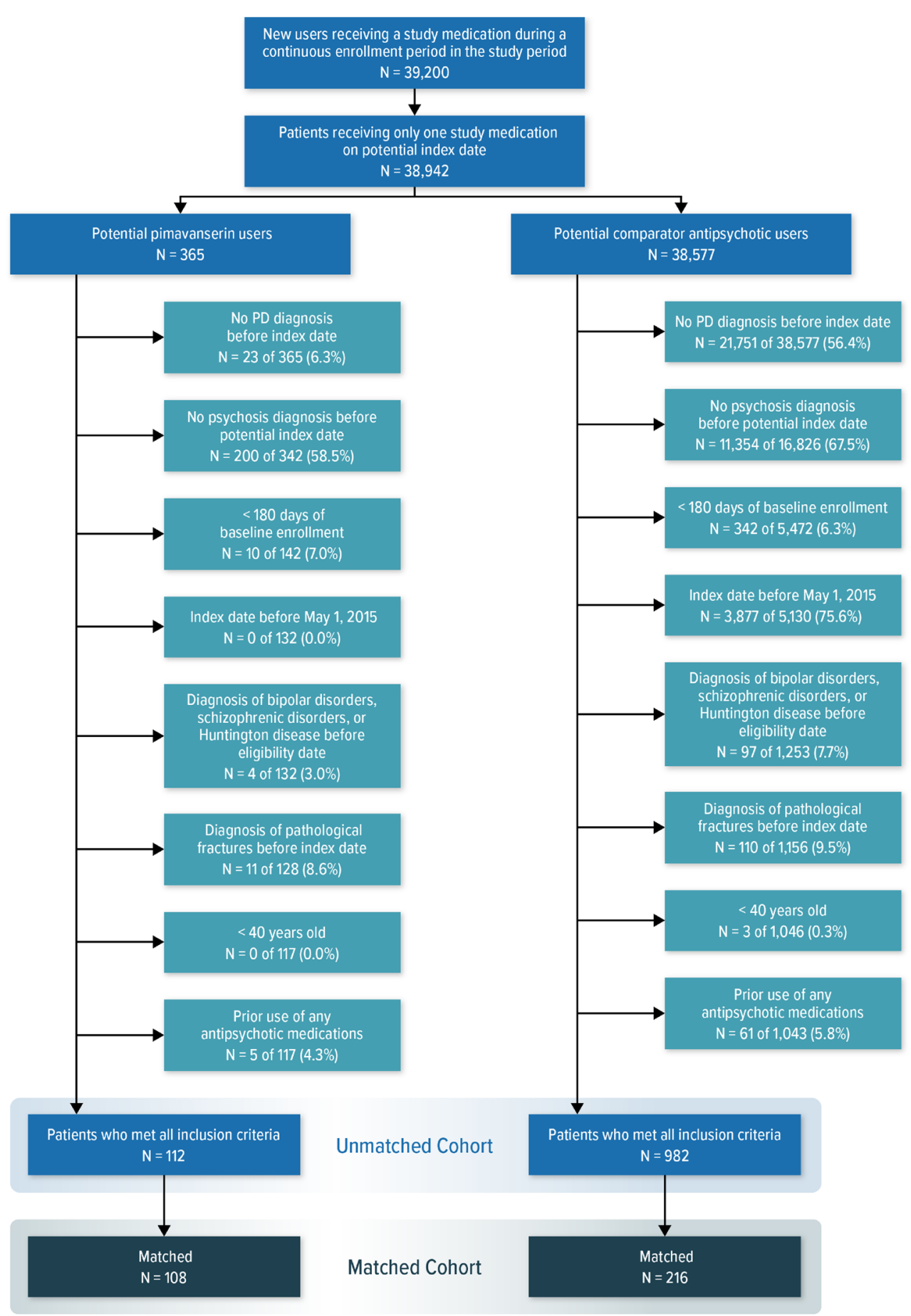

$\mathrm{PD}=$ Parkinson's disease.

Note: Exclusion criteria were applied hierarchically, and patients were counted as excluded at the first exclusion criterion they met. The denominators for percentage excluded updated at each step, as shown.

Fig. 2 Attrition of the study cohort by application of eligibility criteria

pimavanserin initiators and 216 comparator antipsychotic initiators retained in the matched cohort. The characteristics of the matched pimavanserin group remained very similar to those of the unmatched pimavanserin group, as very few pimavanserin users were excluded by the matching. However, much of the comparator group was excluded by the matching (Fig. 2). The characteristics of the matched comparator group differed from the unmatched 


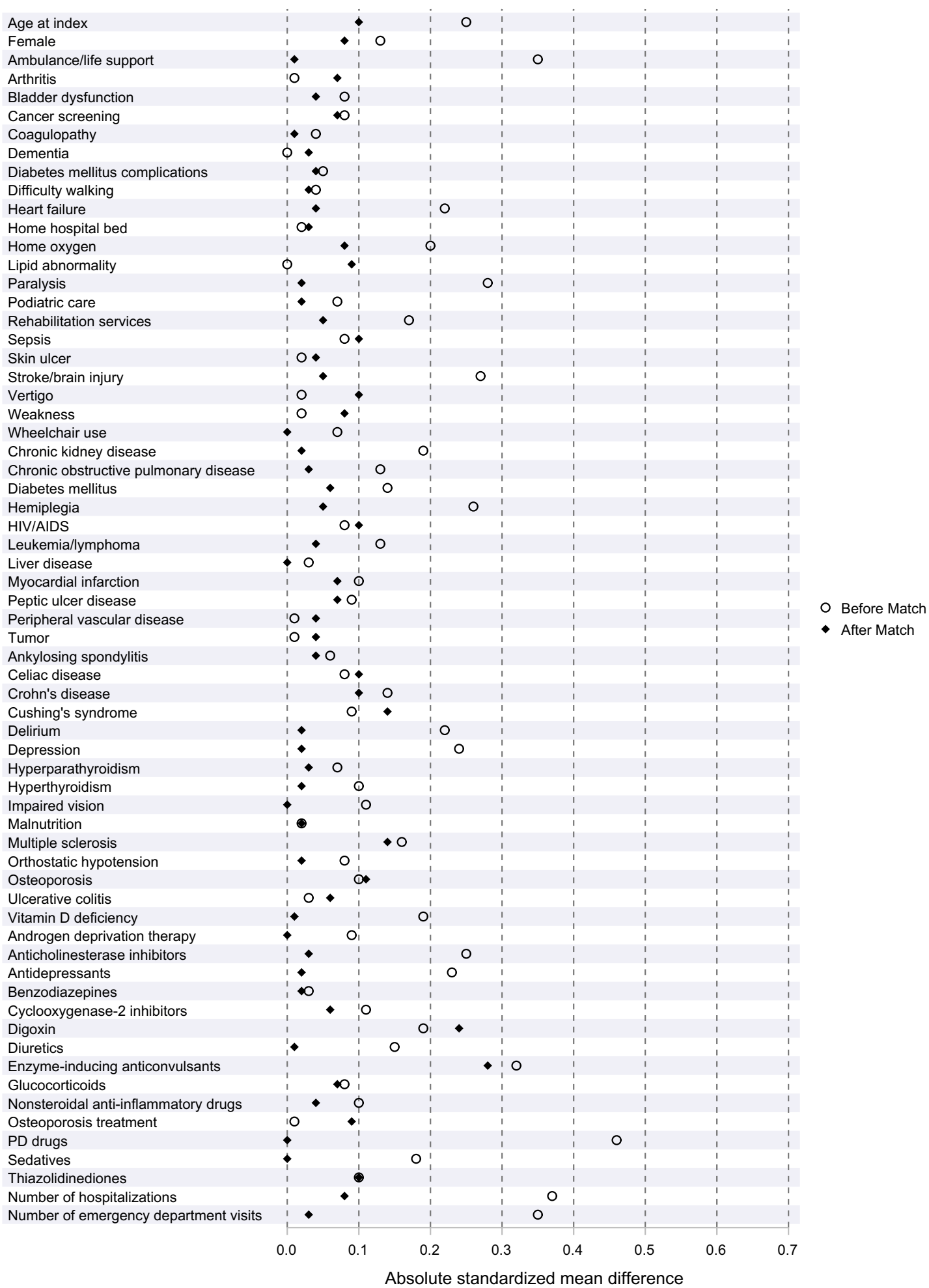

HIVIAIDS = human immunodeficiency virus/acquired immunodeficiency syndrome; PD = Parkinson's disease:

Fig. 3 Relative balance of patient characteristics between the pimavanserin and comparator atypical antipsychotic treatment groups among patients with Parkinson's disease (PD) psychosis, before and after matching 
comparator group (e.g., fewer serious comorbidities than the unmatched group) but were well balanced with the matched pimavanserin group. There were no meaningful differences in patient characteristics remaining in the matched cohort (Fig. 3; Table S5 of the ESM).

The patients in the matched cohort contributed 43 person-years of follow-up in the pimavanserin group and 80 person-years of follow-up in the comparator group. When comparing the matched treatment groups, the incidence rate estimate for the pimavanserin group for composite falls/fractures (18.7 events per 100 person-years; 95\% CI 8.1-36.9) was lower than the rate in the comparator antipsychotic group (26.4 events per 100 person-years; 95\% CI 16.3-40.3), though the small number of cases resulted in a relatively imprecise matched IRR of 0.71 (95\% CI 0.27-1.67) (Table 2). Most of the composite events were falls-very few fracture events were identified-resulting in imprecise incidence rate and IRR estimates for the analyses of overall and site-specific fractures.

When evaluating the incidence of falls and fractures over time during the follow-up, the majority of follow-up time in both groups occurred during the first year after treatment initiation, and very little follow-up time was observed after 1 year. All outcome events in the pimavanserin group were falls that occurred in the first year of follow-up, as were most events in the comparator antipsychotic group (Table S6 of the ESM).

The sensitivity analysis excluding patients with a diagnosis of secondary PD resulted in a matched cohort that included 83 pimavanserin initiators (33 person-years of follow-up) and 166 matched comparators (62 person-years of follow-up) [Fig. S1 of the ESM]. The balance of characteristics between the matched treatment groups was generally good as indicated by the low absolute standardized mean differences (Fig. S2 of the ESM). The number of outcomes identified in this sensitivity analysis was smaller than in the primary analysis, with only 35 fall/fracture events. The resulting IRR estimate of 0.46 (95\% CI 0.17-1.09) was reduced even further than in the primary analysis, though it was imprecise with wide CIs (Table S7 of the ESM).

In the sensitivity analysis where patients were not required to have a psychosis diagnosis before the index date, the sample size was much larger (Fig. S3 of the ESM); the unmatched pimavanserin group contained 276 patients, $40 \%$ of whom had a recorded diagnosis of psychosis, while the comparator group had 2763 patients, 33\% of whom had a psychosis diagnosis (Table S8 of the ESM). The matched cohort for this analysis included 264 pimavanserin (102 person-years of follow-up) and 528 comparator (212 person-years of follow-up) atypical antipsychotic initiators, and the characteristics of these groups were well balanced after matching (Fig. S4 of the ESM). In this sensitivity analysis, the incidence rates of falls/fractures were much higher than those in the primary analysis (Table S9 of the ESM), though the incidence rate was lower in the pimavanserin group than in the comparator group, with a matched IRR estimate of 0.55 (95\% CI 0.34-0.86).

\section{Discussion}

The results of this study did not suggest an increased risk of falls or fractures associated with pimavanserin use compared with other atypical antipsychotics among patients with PDP. The primary and the two sensitivity analyses consistently resulted in IRR point estimates less than 1, though the small size of some analyses resulted in wide CIs.

This study included a comparison group consisting of multiple different atypical antipsychotics of varying potencies, dosage availabilities and ranges, anticholinergic effects, and risk. Some atypical antipsychotics may be associated with developing or worsening movement disorders, and the risk of motor dysfunction may vary by substance [19, 20]; therefore, some of the comparator antipsychotics may not routinely be recommended for use in PD [21, 22]. However, these antipsychotics were used in these patients during the study period, thus we have included them in this analysis profile to evaluate the real-world use of antipsychotics among patients with PDP. Sample sizes were too small to conduct stratified analyses by comparator drug substance.

Antipsychotic medications have previously been associated with an increased risk of falls and fractures [23, 24], and a retrospective chart review of patients prescribed pimavanserin ( $88 \%$ of whom had a PD diagnosis) reported worsening gait instability, which is highly associated with falls, in 5 of 91 patients [25]. However, no studies have been published to date comparing the risk of falls and fractures in patients initiating pimavanserin compared with other atypical antipsychotics. In a randomized placebo-controlled clinical trial of pimavanserin in patients with PDP, falls were among the most common adverse events, although there was no difference in fall risk between the pimavanserin $(11 \%$ of 104 patients) and placebo (9\% of 94 patients) arms in 43 days of follow-up [26]. In addition, a systematic literature review of atypical antipsychotic use in PDP [22] found that adverse events such as falls were reported in four studies comparing pimavanserin with placebo, but there were no differences in falls between the pimavanserin and placebo arms of the studies. This review noted that quetiapine was associated with an increased risk of somnolence, dizziness, and orthostasis, which may all be associated with falls, and olanzapine may exacerbate motor symptoms of PD. The authors of the literature review concluded that pimavanserin should be used as a first-line treatment for PDP because of its risk-benefit profile 
Table 1 Descriptive characteristics of patients with PDP initiating pimavanserin or a comparator antipsychotic medication, unmatched cohort

\begin{tabular}{|c|c|c|c|}
\hline Characteristic & $\begin{array}{l}\text { Overall } \\
N=1094\end{array}$ & $\begin{array}{l}\text { Pimavanserin } \\
N=112\end{array}$ & $\begin{array}{l}\text { Comparator } \\
\text { antipsychotic } \\
N=982\end{array}$ \\
\hline Age at index, mean (SD) & $78.1(9.62)$ & $76.1(8.08)$ & $78.4(9.76)$ \\
\hline Sex, female, $n(\%)$ & $404(36.9)$ & $35(31.3)$ & $369(37.6)$ \\
\hline \multicolumn{4}{|l|}{ Frailty indicators, $n(\%)$} \\
\hline Ambulance/life support & $820(75.0)$ & $68(60.7)$ & $752(76.6)$ \\
\hline Arthritis & $921(84.2)$ & $94(83.9)$ & $827(84.2)$ \\
\hline Bladder dysfunction & $549(50.2)$ & $52(46.4)$ & $497(50.6)$ \\
\hline Cancer screening & $555(50.7)$ & $61(54.5)$ & $494(50.3)$ \\
\hline Coagulopathy & $131(12.0)$ & $12(10.7)$ & $119(12.1)$ \\
\hline Dementia & $967(88.4)$ & $99(88.4)$ & $868(88.4)$ \\
\hline Diabetes mellitus complications & $224(20.5)$ & $21(18.8)$ & $203(20.7)$ \\
\hline Difficulty walking & $895(81.8)$ & $93(83.0)$ & $802(81.7)$ \\
\hline Heart failure & $476(43.5)$ & $38(33.9)$ & $438(44.6)$ \\
\hline Home hospital bed & $120(11.0)$ & $13(11.6)$ & $107(10.9)$ \\
\hline Home oxygen & $55(5.0)$ & $2(1.8)$ & $53(5.4)$ \\
\hline Lipid abnormality & $910(83.2)$ & $93(83.0)$ & $817(83.2)$ \\
\hline Paralysis & $151(13.8)$ & $7(6.3)$ & $144(14.7)$ \\
\hline Podiatric care & 445 (40.7) & $42(37.5)$ & $403(41.0)$ \\
\hline Rehabilitation services & $430(39.3)$ & $36(32.1)$ & $394(40.1)$ \\
\hline Sepsis & $576(52.7)$ & $55(49.1)$ & $521(53.1)$ \\
\hline Skin ulcer & $273(25.0)$ & $27(24.1)$ & $246(25.1)$ \\
\hline Stroke/brain injury & $622(56.9)$ & $50(44.6)$ & $572(58.2)$ \\
\hline Vertigo & $558(51.0)$ & $56(50.0)$ & $502(51.1)$ \\
\hline Weakness & $685(62.6)$ & $71(63.4)$ & $614(62.5)$ \\
\hline Wheelchair use & $181(16.5)$ & $16(14.3)$ & $165(16.8)$ \\
\hline \multicolumn{4}{|c|}{$\begin{array}{l}\text { Additional components of Charlson Comorbidity } \\
\text { Index, } n(\%)\end{array}$} \\
\hline Chronic kidney disease & $273(25.0)$ & $20(17.9)$ & $253(25.8)$ \\
\hline Chronic obstructive pulmonary disease & $421(38.5)$ & $37(33.0)$ & $384(39.1)$ \\
\hline Diabetes mellitus & $416(38.0)$ & $36(32.1)$ & $380(38.7)$ \\
\hline Hemiplegia & $117(10.7)$ & $5(4.5)$ & $112(11.4)$ \\
\hline HIV/AIDS & $3(0.3)$ & 0 & $3(0.3)$ \\
\hline Leukemia/lymphoma & $26(2.4)$ & $1(0.9)$ & $25(2.5)$ \\
\hline Liver disease & $80(7.3)$ & $9(8.0)$ & $71(7.2)$ \\
\hline Myocardial infarction & $151(13.8)$ & $12(10.7)$ & $139(14.2)$ \\
\hline Peptic ulcer disease & $73(6.7)$ & $10(8.9)$ & $63(6.4)$ \\
\hline Peripheral vascular disease & $513(46.9)$ & $52(46.4)$ & $461(46.9)$ \\
\hline Tumor & $279(25.5)$ & $29(25.9)$ & $250(25.5)$ \\
\hline \multicolumn{4}{|l|}{ Other predictors of falls or fractures, $n(\%)$} \\
\hline Ankylosing spondylitis & $72(6.6)$ & $6(5.4)$ & $66(6.7)$ \\
\hline Celiac disease & $3(0.3)$ & 0 & $3(0.3)$ \\
\hline Crohn's disease & $9(0.8)$ & 0 & $9(0.9)$ \\
\hline Cushing's syndrome & $3(0.3)$ & $1(0.9)$ & $2(0.2)$ \\
\hline Delirium & $923(84.4)$ & $86(76.8)$ & $837(85.2)$ \\
\hline Depression & $673(61.5)$ & $57(50.9)$ & $616(62.7)$ \\
\hline Hyperparathyroidism & $27(2.5)$ & $4(3.6)$ & $23(2.3)$ \\
\hline Hyperthyroidism & $46(4.2)$ & $7(6.3)$ & $39(4.0)$ \\
\hline Impaired vision & $50(4.6)$ & $3(2.7)$ & $47(4.8)$ \\
\hline Malnutrition & $373(34.1)$ & $39(34.8)$ & $334(34.0)$ \\
\hline Multiple sclerosis & $13(1.2)$ & 0 & $13(1.3)$ \\
\hline
\end{tabular}


Table 1 (continued)

\begin{tabular}{|c|c|c|c|}
\hline Characteristic & $\begin{array}{l}\text { Overall } \\
N=1094\end{array}$ & $\begin{array}{l}\text { Pimavanserin } \\
N=112\end{array}$ & $\begin{array}{l}\text { Comparator } \\
\text { antipsychotic } \\
N=982\end{array}$ \\
\hline Orthostatic hypotension & $257(23.5)$ & $30(26.8)$ & $227(23.1)$ \\
\hline Osteoporosis & $226(20.7)$ & $19(17.0)$ & $207(21.1)$ \\
\hline Ulcerative colitis & $23(2.1)$ & $2(1.8)$ & $21(2.1)$ \\
\hline Vitamin D deficiency & $342(31.3)$ & $44(39.3)$ & $298(30.3)$ \\
\hline \multicolumn{4}{|l|}{ Comedications, ${ }^{\mathrm{a}} n(\%)$} \\
\hline Androgen deprivation therapy & $3(0.3)$ & $1(0.9)$ & $2(0.2)$ \\
\hline Anticholinesterase inhibitors & $438(40.0)$ & $57(50.9)$ & $381(38.8)$ \\
\hline Antidepressants & $651(59.5)$ & $55(49.1)$ & $596(60.7)$ \\
\hline Benzodiazepines & $383(35.0)$ & $38(33.9)$ & $345(35.1)$ \\
\hline Cyclooxygenase- 2 inhibitors & $23(2.1)$ & $1(0.9)$ & $22(2.2)$ \\
\hline Digoxin & $17(1.6)$ & 0 & $17(1.7)$ \\
\hline Diuretics & $338(30.9)$ & $28(25.0)$ & $310(31.6)$ \\
\hline Enzyme-inducing anticonvulsants & $49(4.5)$ & 0 & $49(5.0)$ \\
\hline Glucocorticoids $^{\mathrm{b}}$ & $120(11.0)$ & $15(13.4)$ & $105(10.7)$ \\
\hline Nonsteroidal anti-inflammatory drugs & $150(13.7)$ & $19(17.0)$ & $131(13.3)$ \\
\hline Osteoporosis treatment & $40(3.7)$ & $4(3.6)$ & $36(3.7)$ \\
\hline PD drugs ${ }^{c}$ & $921(84.2)$ & $108(96.4)$ & $813(82.8)$ \\
\hline Sedatives & $65(5.9)$ & $3(2.7)$ & $62(6.3)$ \\
\hline Thiazolidinediones & $5(0.5)$ & 0 & $5(0.5)$ \\
\hline \multicolumn{4}{|l|}{ Healthcare utilization, mean $(\mathrm{SD})^{\mathrm{d}}$} \\
\hline Number of hospitalizations & $0.6(0.83)$ & $0.3(0.69)$ & $0.6(0.84)$ \\
\hline Number of emergency department visits & $1.9(2.30)$ & $1.2(1.65)$ & $2.0(2.35)$ \\
\hline
\end{tabular}

HIV/AIDS human immunodeficiency virus/acquired immune deficiency syndrome, $P D$ Parkinson's disease, $P D P$ Parkinson's disease-related psychosis, $S D$ standard deviation

Note: All characteristics were assessed during the look-back period using all available time before the index date unless otherwise stated

${ }^{a}$ Assessed in a look-back period of up to 1 year

${ }^{\mathrm{b}}$ Comprised all systemic glucocorticoids (excluded non-systemic administration routes such as topical or inhaled applications)

${ }^{\mathrm{c}}$ Comprised levodopa-carbidopa, anticholinergics, dopamine agonists, monoamine oxidase B inhibitors, catechol-O-methyltransferase inhibitors, amantadine, and istradefylline

${ }^{\mathrm{d}}$ Assessed in the 6 months before the corresponding cohort entry/eligibility date

and ability to improve psychotic symptomatology without worsening motor function.

This study identified pimavanserin users with PD in the time period closely following the introduction of pimavanserin in 2016. Pimavanserin users appeared to be younger and generally healthier, but they had more medication use for PD motor symptoms before antipsychotic initiation than users of comparator antipsychotics, potentially indicating a differing baseline risk for falls; this pattern was consistent with a recent study of pimavanserin users compared with atypical antipsychotic users conducted in US Medicare data [27]. There may be key differences between early users of a more recently introduced medication compared with users of an established class that are difficult to predict [28], including differences in underlying disease state, healthcare access, healthcare-seeking behavior, and quality of care between the treatment groups. Although the effects of residual confounding by unmeasured factors cannot be ruled out in the present study, all measured characteristics were well balanced between the treatment groups after propensity score matching, indicating that confounding due to measured characteristics was well addressed by the study approach.

The results of the present study should be evaluated in view of its potential limitations. This study was conducted with existing administrative claims data, which are generated primarily for billing purposes rather than for clinical diagnoses or research; this may introduce the potential for missing or misclassified study variables, which may potentially change over time because of the transition in coding systems from ICD-9-CM to ICD-10-CM during the study period [8]. The absence of claims for a condition was interpreted as the condition not being present. Falls were 
Table 2 IRs and IRRs of falls and fractures for the unmatched and matched cohorts

\begin{tabular}{llllll}
\hline Outcome & Treatment group & $\begin{array}{l}\text { Number of } \\
\text { patients }\end{array}$ & $\begin{array}{l}\text { Number of } \\
\text { events }\end{array}$ & IR (95\% CI) per 100 PYs & IRR (95\% CI) \\
\hline Unmatched cohort & & & & & \\
Composite falls/fractures & Pimavanserin & 112 & 8 & $17.77(7.67-35.02)$ & $0.44(0.19-0.88)$ \\
& Comparator & $979^{\mathrm{a}}$ & 173 & $40.82(34.97-47.38)$ & Reference \\
Falls & Pimavanserin & 112 & 8 & $17.77(7.67-35.02)$ & $0.48(0.20-0.97)$ \\
& Comparator & $979^{\mathrm{a}}$ & 157 & $37.02(31.46-43.29)$ & Reference \\
Any fracture & Pimavanserin & 112 & 1 & $2.21(0.06-12.34)$ & $0.33(0.01-1.97)$ \\
& Comparator & 982 & 29 & $6.80(4.55-9.76)$ & Reference \\
Matched cohort & & & & $18.74(8.09-36.93)$ & $0.71(0.27-1.67)$ \\
Composite falls/fractures & Pimavanserin & 108 & 8 & $26.38(16.33-40.32)$ & Reference \\
& Comparator & $215^{\mathrm{a}}$ & 21 & $18.74(8.09-36.93)$ & $0.88(0.33-2.15)$ \\
Falls & Pimavanserin & 108 & 8 & $21.34(12.43-34.16)$ & Reference \\
Any fracture & Comparator & $215^{\mathrm{a}}$ & 17 & $2.34(0.06-13.01)$ & $0.31(0.01-2.56)$ \\
& Pimavanserin & 108 & 1 & $7.50(2.75-16.33)$ & Reference \\
\hline
\end{tabular}

$C I$ confidence interval, $I R$ incidence rate, IRR incidence rate ratio, $P Y s$ person-years

${ }^{a}$ Sample sizes at the index date were reduced because of fall events occurring before the index date, resulting in patients being not at risk at the beginning of the follow-up

Fig. 4 Propensity score distributions of the pimavanserin and comparator atypical antipsychotic treatment groups among patients with Parkinson's disease psychosis

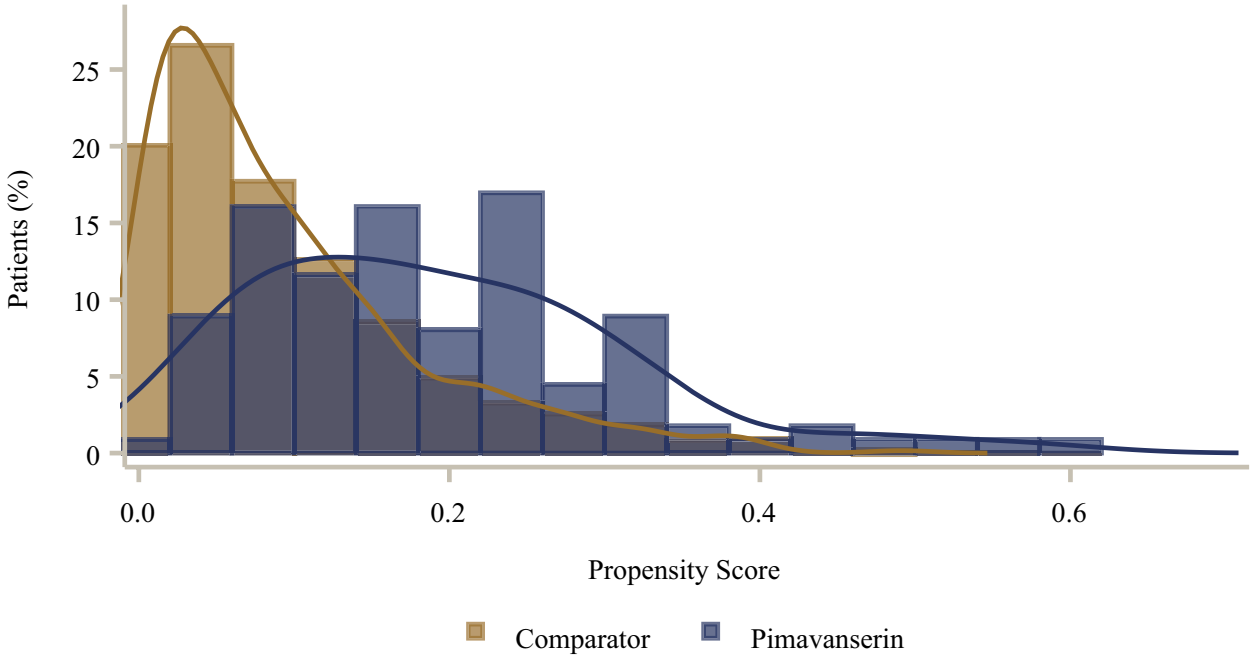

identified with diagnosis codes alone, though not all falls may be medically attended; thus, only more severe falls may be identified with diagnosis codes. Similarly, we required both fracture diagnosis and procedure codes (e.g., reduction or repositioning with open, closed, percutaneous, or percutaneous endoscopic approaches) to ensure identification of newly treated fractures, though not all fractures may require repair or be repaired immediately, potentially resulting in undercounting of fracture events. Additionally, while we categorized all fractures by site, not all diagnosis codes used in the study specified laterality, thus we could not differentiate between site-specific fractures occurring on opposite body sides (e.g., between a left and right lower limb), potentially resulting in undercounting of fracture events. Because of the relatively small number of fractures identified in the study, estimates of individual fracture sites were imprecise and relatively uninformative. Patients with evidence of pathologic fractures were excluded or censored, but claims data may lack the granularity to determine etiology of all fracture events or differentiate accidental falls/fractures from those with external causes. In addition, pharmacy claims for medications indicate that a prescription has been dispensed, but it may not reflect actual use of the medication by the patient. These insurance claims do not contain information on patient death; while death would result in disenrollment from the database, mortality status or cause of death cannot 
be evaluated in these data. Last, this study used data from those with employer-based commercial insurance or supplementary Medicare plans; while these databases are large, they may not be generally representative of all patients with PDP, including those without insurance, with Medicaid coverage, or with other non-employer-sponsored insurance coverage.

Sensitivity analyses evaluated the impact of different eligibility criteria, as PD and psychosis may be slow or difficult to diagnose and may not be recorded reliably in claims data. All patients met a relatively strict definition of PD based on inpatient diagnoses, outpatient diagnoses, or pharmacy medication claims - similar definitions have been shown to have very good validity [29]; however, we excluded patients with a recorded diagnosis of secondary PD in a sensitivity analysis to reduce potential misclassification of PD. Previous researchers have applied a similar exclusion criterion when attempting to compare patients with and without PD [1], although both treatment groups in the current study comprised patients with PD being treated for PDP. In this sensitivity analysis, the exclusion criterion of secondary PD disproportionately excluded patients in the pimavanserin group (20.5\% of identified pimavanserin users with a PD diagnosis vs $13.3 \%$ of comparators with a PD diagnosis), which was surprising given that receiving a prescription of pimavanserin from a specialty pharmacy required healthcare providers to submit a treatment form confirming a diagnosis of PDP. While misclassification of PD in pimavanserin users seems unlikely, pimavanserin users were more likely to have diagnoses of PD-related symptoms and more PD medication use, thus the diagnoses of secondary PD may have been part of a diagnostic workup before arriving at a final diagnosis of PD.

The identification of a clinical diagnosis of psychosis might be challenging in an administrative claims database using coded diagnoses, [7, 9] and it has been reported that recorded psychosis diagnoses may have decreased after transitioning to ICD-10-CM coding systems [8]; thus, a recorded diagnosis of psychosis was not required in an additional sensitivity analysis where the presence of PDP was inferred by the use of antipsychotic medications among patients with PD. This analysis resulted in a larger sample size, and it may have included additional patients with true PDP but who lacked a recorded psychosis diagnosis in the available data before antipsychotic treatment initiation. The incidence rates of falls and fractures were higher in the treatment groups when a diagnosis of psychosis was not required, perhaps because of the inclusion of some patients using antipsychotics off-label for treatment of non-psychosis symptoms. Related research of pimavanserin use in PDP has not required patients to have a psychosis diagnosis [27, 30], but antipsychotics may frequently be used for the treatment of behavioral symptoms of dementia, sleep disorders, or other non-psychosis conditions [31]; thus, this sensitivity analysis may have also included additional patients using antipsychotics without true psychosis. Because of prescribing restrictions on pimavanserin, misclassified psychosis status may be more common in the comparator group. The combination of inconsistent recording of psychosis symptoms in claims data with the relatively common off-label use of antipsychotics for non-psychosis symptoms may indicate that the primary analysis requiring a psychosis diagnosis was overly restrictive, while the sensitivity analysis not requiring a psychosis diagnosis was overly broad. However, the relative consistency of the IRR estimates from these analyses is reassuring.

\section{Conclusions}

Results of this study do not suggest an increased risk of falls or fractures among patients with PDP taking pimavanserin compared with those taking other atypical antipsychotics, and the sensitivity analyses suggest a decreased risk of falls/ fractures. While small sample sizes resulted in imprecision in some estimates, IRR estimates were consistently below the null across all analyses. Additional research is warranted when pimavanserin is more widely used among patients with PDP.

Supplementary Information The online version contains supplementary material available at https://doi.org/10.1007/s40801-021-00284-1.

Acknowledgements The authors acknowledge the editorial assistance of Paul Hobson of RTI Health Solutions.

\section{Declarations}

Funding This work was funded by Acadia Pharmaceuticals.

Conflicts of interest/Competing interests $\mathrm{CD}$ and MET are employees of Acadia Pharmaceuticals, which produces one of the medications evaluated in this study. GD was an employee of Acadia Pharmaceuticals while this work was performed. JBL, JF, JLB, MSA, and HED are employees of RTI Health Solutions. MER was an employee of RTI Health Solutions while this work was performed. This study was conducted by RTI Health Solutions with funding from Acadia Pharmaceuticals under a contract that included independent publication rights.

Ethics approval This analysis of deidentified data was exempted from review by the Institutional Review Board of RTI International.

Consent to participate Individual-level consent was not required for this analysis using deidentified secondary data.

Consent for publication Individual-level consent was not required for this analysis of deidentified secondary data.

Availability of data and material The data that support the findings of this study were licensed from the IBM MarketScan Research Databases. These data are not publicly available. Any researcher requiring access to the raw data that were used to generate the analytical files can 
access the data under a license agreement, including the payment of appropriate license fees, between that third party and IBM.

Code availability Researchers seeking to access the analytic code used in the analysis of this data may contact the study team.

Authors' contributions JBL: conceptualization, methodology, visualization, writing (original draft); JF: conceptualization, methodology, visualization, writing (original draft); $\mathrm{CD}$ : conceptualization, writing (review and editing); MET: conceptualization, funding acquisition, writing (review and editing); JLB: methodology, software, formal analysis, data curation, writing (review and editing); MSA: conceptualization, supervision, funding acquisition, writing (review and editing); HED: resources, project administration, writing (review and editing); MER: conceptualization, methodology, resources, visualization, supervision, project administration, funding acquisition, writing (review and editing); GD: conceptualization, writing (review and editing).

Open Access This article is licensed under a Creative Commons Attribution-NonCommercial 4.0 International License, which permits any non-commercial use, sharing, adaptation, distribution and reproduction in any medium or format, as long as you give appropriate credit to the original author(s) and the source, provide a link to the Creative Commons licence, and indicate if changes were made. The images or other third party material in this article are included in the article's Creative Commons licence, unless indicated otherwise in a credit line to the material. If material is not included in the article's Creative Commons licence and your intended use is not permitted by statutory regulation or exceeds the permitted use, you will need to obtain permission directly from the copyright holder. To view a copy of this licence, visit http://creativecommons.org/licenses/by-nc/4.0/.

\section{References}

1. Kalilani L, Asgharnejad M, Palokangas T, Durgin T. Comparing the incidence of falls/fractures in Parkinson's disease patients in the US population. PLoS ONE. 2016;11(9): e0161689. https://doi. org/10.1371/journal.pone.0161689.

2. Kianirad Y, Simuni T. Pimavanserin, a novel antipsychotic for management of Parkinson's disease psychosis. Expert Rev Clin Pharmacol. 2017;10(11):1161-8. https://doi.org/10.1080/17512 433.2017.1369405.

3. Forns J, Layton JB, Bartsch J, Turner ME, Dempsey C, Anthony $\mathrm{M}$, et al. Increased risk of falls and fractures in patients with psychosis and Parkinson disease. PLoS ONE. 2021;16(1): e0246121. https://doi.org/10.1371/journal.pone.0246121.

4. Nuplazid (pimavanserin) prescribing information. San Diego (CA): Acadia Pharmaceuticals Inc.; 2019. https://www.acces sdata.fda.gov/drugsatfda_docs/label/2019/207318s009,21079 3s003lbl.pdf. Accessed 18 Nov 2019.

5. U.S. Centers for Medicare \& Medicaid Services. 2018 ICD$10 \mathrm{CM}$ and GEMs. 11 August 2017, 2017. https://www.cms. gov/Medicare/Coding/ICD10/2018-ICD-10-CM-and-GEMs. Accessed 25 Aug 2021.

6. Nam YH, Mendelsohn AB, Panozzo CA, Maro JC, Brown JS. Health outcomes coding trends in the US Food and Drug Administration's Sentinel System during transition to International Classification of Diseases-10 coding system: a brief review. Pharmacoepidemiol Drug Saf. 2021;30(7):838-42. https://doi.org/10.1002/pds.5216.

7. Kurdyak P, Lin E, Green D, Vigod S. Validation of a population-based algorithm to detect chronic psychotic illness. Can
J Psychiatry. 2015;60(8):362-8. https://doi.org/10.1177/07067 4371506000805.

8. Stewart CC, Lu CY, Yoon TK, Coleman KJ, Crawford PM, Lakoma MD, et al. Impact of ICD-10-CM transition on mental health diagnoses recording. EGEMS (Wash DC). 2019;7(1):14. https://doi.org/10.5334/egems.281.

9. Hamedani AG, Weintraub D, Willis AW. Medicare claims data underestimate hallucinations in older adults with dementia. Am J Geriatr Psychiatry. 2021. https://doi.org/10.1016/j.jagp.2021. 07.018.

10. Cuthbertson CC, Kucharska-Newton A, Faurot KR, Sturmer $\mathrm{T}$, Jonsson Funk M, Palta P, et al. Controlling for frailty in pharmacoepidemiologic studies of older adults: validation of an existing Medicare claims-based algorithm. Epidemiology. 2018;29(4):556-61. https://doi.org/10.1097/EDE.0000000000 000833.

11. Faurot KR, Jonsson Funk M, Pate V, Brookhart MA, Patrick A, Hanson LC, et al. Using claims data to predict dependency in activities of daily living as a proxy for frailty. Pharmacoepidemiol Drug Saf. 2015;24(1):59-66. https://doi.org/10.1002/pds. 3719.

12. Deyo RA, Cherkin DC, Ciol MA. Adapting a clinical comorbidity index for use with ICD-9-CM administrative databases. J Clin Epidemiol. 1992;45(6):613-9. https://doi.org/10.1016/08954356(92)90133-8.

13. Sun JW, Rogers JR, Her Q, Welch EC, Panozzo CA, Toh S, et al. Adaptation and validation of the combined comorbidity score for ICD-10-CM. Med Care. 2017;55(12):1046-51. https://doi.org/10. 1097/MLR.0000000000000824.

14. Austin PC. Balance diagnostics for comparing the distribution of baseline covariates between treatment groups in propensity-score matched samples. Stat Med. 2009;28(25):3083-107. https://doi. org/10.1002/sim.3697.

15. Parsons L. Performing a $1: \mathrm{N}$ case-control match on propensity score. In: SAS Global Forum Proceedings / SUGI 29 2004. 2004. https://support.sas.com/resources/papers/proceedings/proce edings/sugi29/165-29.pdf. Accessed 18 Oct 2021.

16. Austin PC. Optimal caliper widths for propensity-score matching when estimating differences in means and differences in proportions in observational studies. Pharm Stat. 2011;10(2):150-61. https://doi.org/10.1002/pst.433.

17. Dobson AJ, Kuulasmaa K, Eberle E, Scherer J. Confidence intervals for weighted sums of Poisson parameters. Stat Med. 1991;10(3):457-62. https://doi.org/10.1002/sim.4780100317.

18. Sahai H, Khurshid A. Statistics and epidemiology: methods, techniques, and applications. Boca Raton: CRC Press LLC; 1996.

19. Martino D, Karnik V, Osland S, Barnes TRE, Pringsheim TM. Movement disorders associated with antipsychotic medication in people with schizophrenia: an overview of Cochrane reviews and meta-analysis. Can J Psychiatry. 2018;63(11):706743718777392. https://doi.org/10.1177/0706743718777392.

20. Chyou TY, Nishtala R, Nishtala PS. Comparative risk of Parkinsonism associated with olanzapine, risperidone and quetiapine in older adults: a propensity score matched cohort study. Pharmacoepidemiol Drug Saf. 2020;29(6):692-700. https://doi.org/10. 1002/pds.5007.

21. Divac N, Stojanovic R, Savic Vujovic K, Medic B, Damjanovic A, Prostran M. The efficacy and safety of antipsychotic medications in the treatment of psychosis in patients with Parkinson's disease. Behav Neurol. 2016;2016:4938154. https://doi.org/10.1155/2016/ 4938154.

22. Zhang H, Wang L, Fan Y, Yang L, Wen X, Liu Y, et al. Atypical antipsychotics for Parkinson's disease psychosis: a systematic review and meta-analysis. Neuropsychiatr Dis Treat. 2019;15:2137-49. https://doi.org/10.2147/NDT.S201029. 
23. Lee SH, Hsu WT, Lai CC, Esmaily-Fard A, Tsai YW, Chiu CC, et al. Use of antipsychotics increases the risk of fracture: a systematic review and meta-analysis. Osteoporos Int. 2017;28(4):116778. https://doi.org/10.1007/s00198-016-3881-3.

24. Fraser LA, Liu K, Naylor KL, Hwang YJ, Dixon SN, Shariff SZ, et al. Falls and fractures with atypical antipsychotic medication use: a population-based cohort study. JAMA Intern Med. 2015;175(3):450-2. https://doi.org/10.1001/jamainternmed.2014.6930.

25. Sellers J, Darby RR, Farooque A, Claassen DO. Pimavanserin for psychosis in Parkinson's disease-related disorders: a retrospective chart review. Drugs Aging. 2019;36(7):647-53. https://doi.org/10. 1007/s40266-019-00655-y.

26. Cummings J, Isaacson S, Mills R, Williams H, Chi-Burris K, Corbett A, et al. Pimavanserin for patients with Parkinson's disease psychosis: a randomised, placebo-controlled phase 3 trial. Lancet. 2014;383(9916):533-40. https://doi.org/10.1016/S0140-6736(13) 62106-6.

27. Mosholder A, Ma Y, Akhtar S, Podskalny G, Wei Y, Liao J, et al. Abstracts of the MDS Virtual Congress 2020. Mov Disord. 2020;35 Suppl. 1(S1):S1-702. https://doi.org/10.1002/mds.28268.
28. Gagne JJ, Bykov K, Willke RJ, Kahler KH, Subedi P, Schneeweiss $\mathrm{S}$. Treatment dynamics of newly marketed drugs and implications for comparative effectiveness research. Value Health. 2013;16(6):1054-62. https://doi.org/10.1016/j.jval.2013.05.008.

29. Butt DA, Tu K, Young J, Green D, Wang M, Ivers N, et al. A validation study of administrative data algorithms to identify patients with Parkinsonism with prevalence and incidence trends. Neuroepidemiology. 2014;43(1):28-37. https://doi.org/10.1159/00036 5590.

30. Hwang YJ, Alexander GC, An H, Moore TJ, Mehta HB. Risk of hospitalization and death associated with pimavanserin use in older adults with Parkinson disease. Neurology. 2021. https://doi. org/10.1212/WNL.0000000000012601.

31. Carton L, Cottencin O, Lapeyre-Mestre M, Geoffroy PA, Favre J, Simon N, et al. Off-label prescribing of antipsychotics in adults, children and elderly individuals: a systematic review of recent prescription trends. Curr Pharm Des. 2015;21(23):3280-97. https:// doi.org/10.2174/1381612821666150619092903.

\section{Authors and Affiliations}

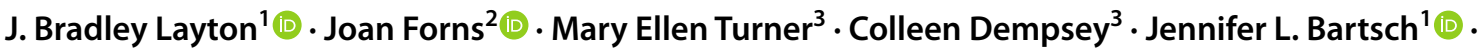

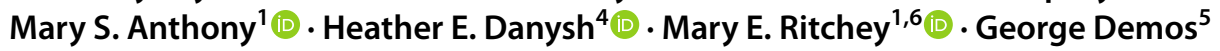

\author{
Joan Forns \\ jforns@ rti.org \\ Mary Ellen Turner \\ meturner@ACADIA-Pharm.com \\ Colleen Dempsey \\ cdempsey@ACADIA-Pharm.com \\ Jennifer L. Bartsch \\ jbartsch@rti.org \\ Mary S. Anthony \\ manthony@rti.org \\ Heather E. Danysh \\ hdanysh@rti.org \\ Mary E. Ritchey \\ marybeth@medtechepi.com
}

George Demos

gdemos@ACADIA-Pharm.com

1 RTI Health Solutions, Post Office Box 12194, Research Triangle Park, NC 27709-2194, USA

2 RTI Health Solutions, Barcelona, Spain

3 Acadia Pharmaceuticals, Inc., Princeton, NJ, USA

4 RTI Health Solutions, Waltham, MA, USA

5 Present Address: Acadia Pharmaceuticals, Inc., San Diego, CA, USA

6 Present Address: Med Tech Epi, LLC, and Rutgers University Center for Pharmacoepidemiology and Treatment Science, New Brunswick, USA 